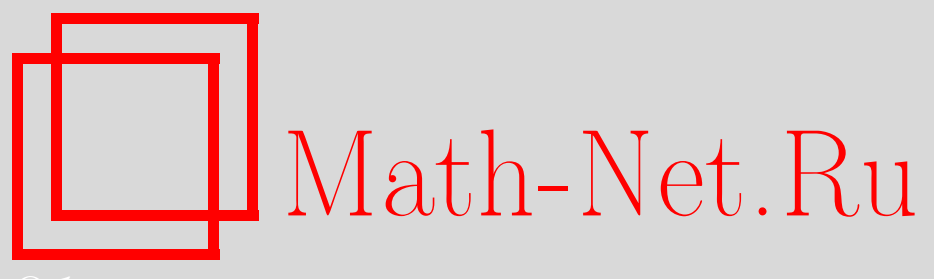

А. А. Боровков, Переходные явления для случайных блужданий с разнораспределенными скачками, имеющими бесконечные дисперсии, Теория вероятн. и ее примен., 2005, том 50, выпуск 2, 224-240

DOI: https://doi.org/10.4213/tvp105

Использование Общероссийского математического портала Math-Net.Ru подразумевает, что вы прочитали и согласны с пользовательским соглашением

http://www . mathnet.ru/rus/agreement

Параметры загрузки:

IP : 54.80 .73 .141

26 апреля 2023 г., 15:17:29

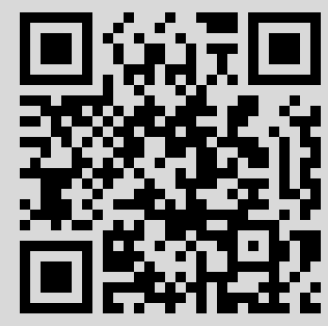




\section{ПЕРЕХОДНЫЕ ЯВЛЕНИЯ ДЛЯ СЛУЧАЙНЫХ БЛУЖДАНИЙ С РАЗНОРАСПРЕДЕЛЕННЫМИ СКАЧКАМИ, ИМЕЮЩИМИ БЕСКОНЕЧНЫЕ ДИСПЕРСИИ}

Пусть $\zeta_{1}, \zeta_{2}, \ldots$ - независимые случайные величины,

$$
Z_{n}=\sum_{i=1}^{n} \zeta_{i}, \quad \bar{Z}_{n}=\max _{k \leqslant n} Z_{k}, \quad Z=\bar{Z}_{\infty} .
$$

Хорошо известно, что если $\zeta_{i}$ одинаково распределены, то $Z$ есть собственная случайная величина при $\mathbf{E} \zeta_{i}=-a<0$ и $Z=\infty$ п.н., если $a=0$. Предельное распределение $\bar{Z}_{n}$ при $n \rightarrow \infty, a \rightarrow 0$ (в схеме серий) и $\mathbf{E} \zeta_{i}^{2}<\infty$ изучено достаточно полно (см., например, [1]-[3]).

В работе изучается предельное распределение $\bar{Z}_{n}$ при $\mathbf{E} \zeta_{i} \rightarrow 0$, $n \rightarrow \infty$, в случае, когда $\mathbf{E} \zeta_{i}^{2}=\infty$, а слагаемые $\zeta_{i}$ являются разнораспределенными.

Ключевые слова и фразы: случайные блуждания, максимум сумм, переходные явления, сходимость к устойчивым процессам, разнораспределенные слагаемые, бесконечная дисперсия.

1. Введение. Под переходными явлениями в задачах о пересечении границы траекторией случайного блуждания обычно понимают следующее. Пусть $\zeta, \zeta_{1}, \zeta_{2}, \ldots$ - независимые одинаково распределенные случайные величины,

$$
Z_{n}=\sum_{i=1}^{n} \zeta_{i}, \quad \bar{Z}_{n}=\max _{k \leqslant n} Z_{k}, \quad Z=\bar{Z}_{\infty} .
$$

Хорошо известно, что если $\mathbf{E} \zeta=-a<0$, то $Z$ есть собственная случайная величина, и $Z=\infty$ п.н., если $a=0$. Переходными называют явления, возникающие при изучении распределения $Z$ (или максимума конечного числа сумм), когда $0<a \rightarrow 0$. Чтобы сделать постановку задачи более точной, надо ввести в рассмотрение схему серий, когда

* Институт математики им. С.Л. Соболева СО РАН, просп. Академика Коптюга, 4, 630090 Новосибирск, Россия; e-mail: borovkov@math.nsc.ru

1) Работа выполнена при поддержке Российского фонда фундаментальных исследований (проект № 02-01-00902) и INTAS (проект № 03-51-5018). 
распределение $\zeta$ зависит от некоторого меняющегося параметра, который в нашем случае может быть отождествлен с $a$, и рассматривать ситуацию, когда $a \rightarrow 0$. Иногда бывает удобным рассматривать и традиционную схему серий, т.е. последовательности $\zeta_{1, n}, \zeta_{2, n}, \ldots$, где распределение $\zeta_{k, n}$ зависит от номера серии $n$. В этом случае $a=a(n)$ зависит от $n$ таким образом, что $a(n) \rightarrow 0$ при $n \rightarrow \infty$. Однако при изучении распределения $Z$ введение схемы серий с параметром $n$ может оказаться искусственным, так как этот параметр в постановке задачи может вообще не участвовать. Поскольку, кроме того, все нормирующие множители в последующем будут функциями от $a$ (а не от $n$ ), то часто первый подход к введению схемы серий (с помощью параметра $a$ ) представляется более предпочтительным.

В дальнейшем мы будем считать, что имеет место схема серий, в которой распределение $\zeta=\zeta^{(a)}$ зависит от $a$, но индекс $(a)$, указывающий на эту зависимость, будем для краткости опускать.

Далее, нам будет удобнее иметь дело с центрированными случайными величинами $\xi_{i}=\zeta_{i}+a$, так что $\mathbf{E} \xi_{i}=0$. Тогда изучаемый функционал $Z$ от последовательности сумм $Z_{k}$ будет иметь вид

$$
Z=\bar{S}(a)=\sup _{k \geqslant 0}\left(S_{k}-a k\right), \quad S_{n}=\sum_{i=1}^{n} \xi_{i} .
$$

Мы будем рассматривать также максимумы конечного числа сумм

$$
\bar{S}_{n}(a)=\max _{k \leqslant n}\left(S_{k}-a k\right) .
$$

Изучение переходных явлений во многом стимулировалось приложениями. В задачах, связанных с системами обслуживания, задача о переходных явлениях возникает в ситуации, когда система работает в условиях «тяжелой нагрузки», т.е. в случае, когда интенсивность входного потока требований близка к максимально возможной интенсивности обслуживания. В этом случае - например, для простейшей одноканальной системы обслуживания, длина очереди (или время обслуживания) в которой в момент $n$ может быть описана с помощью случайной величины $\bar{S}_{n}(a)$, - мы будем иметь ситуацию, когда $a$ мало.

Известно, что если $\mathbf{E} \xi^{2}=\sigma_{a}^{2} \rightarrow \sigma^{2}<\infty$ при $a \rightarrow 0$, то для $n=T a^{-2}$, где $T \leqslant \infty$ фиксировано, сушествует предельное распределение $a \bar{S}_{n}(a)$ :

$$
\lim _{a \rightarrow 0} \mathbf{P}\left\{a \bar{S}_{n}(a)>z\right\}=\mathbf{P}\left\{\max _{t \leqslant T}(\sigma w(t)-t)>z\right\},
$$

где $w(t)$ - стандартный винеровский процесс. В частности, при $T=\infty$ правая часть в $(1)$ превращается в $e^{-(2 z) / \sigma^{2}}$. Подробнее об этом см., например, [1]-[3].

В случае $\mathbf{E} \xi^{2}=\infty$ нам известны лишь некоторые частные результаты, относящиеся к предельному распределению $Z$ для специальных 
типов распределений $\zeta$, см. [4]-[6]. Эти результаты не позволяют понять природу предельных распределений в общем случае. Аналитические методы исследования в названных работах, связанные с факторизационными тождествами, обладают известной ограниченностью, что делает рассматриваемые задачи в их более общей постановке труднодоступными для этих методов.

2. Основные предельные теоремы о сходимости к устойчивым процессам. Мы будем рассматривать более общую постановку задачи, чем описанная во введении. Именно, мы будем предполагать, что слагаемые $\zeta_{i}$ являются разнораспределенными независимыми случайными величинами в схеме серий, при этом

$$
\mathbf{E}\left|\zeta_{i}\right|<\infty, \quad \text { но } \quad \max _{i} \mathbf{E} \zeta_{i}^{2}=\infty \text {. }
$$

В первых двух теоремах, приведенных ниже и относящихся к предельному распределению $Z_{n}$ при $n \rightarrow \infty$, нам будет удобнее в качестве параметра схемы серий выбрать $n$. В последующих утверждениях параметр схемы серий будет заменен на значение $a \rightarrow 0$, ассоциированное со средними $n^{-1} \sum_{j=1}^{n} a_{j} \rightarrow 0$, а под $n$ мы будем понимать функцию $n=n(a) \uparrow \infty$ при $a \downarrow 0$. В теореме 3 она будет конкретизирована.

В случае бесконечных вторых моментов величин $\zeta_{i}$ отыскание предельных распределений $Z_{n}, \bar{Z}_{n}$ возможно лишь в случае, когда распределения сумм $S_{n}=\sum_{i=1}^{n} \xi_{i}$ центрированных случайных величин $\xi_{i}=\zeta_{i}+a_{i}$, $a_{i}=-\mathbf{E} \zeta_{i}$, сходятся (после соответствующей нормировки) к устойчивому закону, а средние значения $-a_{i}$ ведут себя достаточно правильным образом. Это означает, что мы должны предполагать выполненными условия, которые обеспечивали бы такие сходимость и правильность.

Введем в рассмотрение нужные нам обозначения и условия. Пусть $\mathbf{F}_{j}$ - распределение случайной величины $\xi_{j}$. Почти все накладываемые ниже условия будут относиться к «усредненным» распределениям

$$
\mathbf{F}=\frac{1}{n} \sum_{j=1}^{n} \mathbf{F}_{j}
$$

при $n$, выбранном подходящим образом. Положим

$$
\begin{aligned}
V_{j}(t) & =\mathbf{F}_{j}((t, \infty)), \quad W_{j}(t)=\mathbf{F}_{j}((-\infty,-t)), \\
\mathbf{F}_{j}(t) & =V_{j}(t)+W_{j}(t)=\mathbf{P}\left\{\left|\xi_{j}\right|>t\right\}, \\
V(t) & =\frac{1}{n} \sum_{j=1}^{n} V_{j}(t), \quad W(t)=\frac{1}{n} \sum_{j=1}^{n} W_{j}(t), \quad F(t)=V(t)+W(t) .
\end{aligned}
$$

Функции $V, W, F$ будем называть усредненными хвостами. Там, где надо будет подчеркнуть их зависимость от $n$, мы будем писать $V^{(n)}, W^{(n)}, F^{(n)}$ соответственно. 
Мы будем предполагать, что усредненные хвосты $F(t)$ удовлетворяют следуюшим условиям «равномерного правильного изменения» [UR] (uniform regularity).

[UR]. Существует фиксированная (не зависящая от параметра схемы серий) правильно меняющаяся функция $G(t)=t^{-\alpha} L(t)(\alpha \in(1,2)$, $L$ - медленно меняющаяся функиия) такая, что

1)

$$
\lim _{t \rightarrow \infty, n \rightarrow \infty} \frac{F^{(n)}(t)}{G(t)}=1,
$$

другими словами, для любого заданного $\delta>0$ найдутся $n_{\delta}, t_{\delta}$, для коmopblx

2)

$$
\begin{gathered}
\sup _{n \geqslant n_{\delta}, t \geqslant t_{\delta}}\left|\frac{F^{(n)}(t)}{G(t)}-1\right|<\delta ; \\
\lim _{t \rightarrow \infty, n \rightarrow \infty} \frac{V^{(n)}(t)}{G(t)}=\rho_{+} \in[0,1] .
\end{gathered}
$$

Из последнего условия следует, что существует также

$$
\lim _{t \rightarrow \infty, n \rightarrow \infty} \frac{W^{(n)}(t)}{G(t)}=\rho_{-}=1-\rho_{+} \in[0,1] .
$$

Везде в дальнейшем мы будем предполагать, что

$$
\mathbf{E} \xi_{j}=0 .
$$

Мы можем сформулировать теперь основную предельную теорему о сходимости к устойчивому закону, которую мы будем использовать. Положим

$$
X_{n}=\frac{S_{n}}{b(n)}, \quad b(n)=G^{(-1)}\left(\frac{1}{n}\right)=\inf \left\{t: G(t) \leqslant \frac{1}{n}\right\}
$$

и обозначим через [S] условие «пренебрежимой малости» слагаемых $\xi_{j} / b(n)$.

[S]. Дия ююбого $\varepsilon>0$

$$
\max _{j \leqslant n} \mathbf{P}\left\{\left|\xi_{j}\right|>\varepsilon b(n)\right\}=\max _{j \leqslant n} G_{j}(\varepsilon b(n)) \longrightarrow 0
$$

npu $n \rightarrow \infty$.

Теорема 1. Пусть усредненнье хвость $F^{(n)}$ удовлетворяют условиям [UR]. Тогда при выполнении [S] и при $n \rightarrow \infty$ распределения $X_{n}$ слабо сходлтся $\kappa$ устойчивому распределению $\mathrm{F}^{(\alpha, \rho)}: X_{n} \Rightarrow X^{(\alpha, \rho)}$, $\rho=\rho_{+}-\rho_{-}$, где $X^{(\alpha, \rho)}$ имеет характеристическую функцию

$$
\begin{gathered}
\varphi^{(\alpha, \rho)}(t)=\mathbf{E} e^{i t X^{(\alpha, \rho)}}=\exp \left\{|t|^{\alpha} B(\alpha, \rho, \phi)\right\}, \quad \phi=\operatorname{sign} t, \\
B(\alpha, \rho, \phi)=\frac{\Gamma(2-\alpha)}{1-\alpha}\left[i \rho \phi \sin \frac{\alpha \pi}{2}-\cos \frac{\alpha \pi}{2}\right] .
\end{gathered}
$$


Д о к а з а т е л ь с т в о. Воспользуемся общей теоремой о сходимости к безгранично делимым законам (см., например, [7]). В наших условиях эта теорема приобретает следуюший вид.

Пусть $\mathbf{E} \xi_{j}=0$ и выполнено условие [S]. Для того чтобы при $n \rightarrow \infty$ имела место сходимость

$$
X_{n} \Rightarrow X^{(\alpha, \rho)}, \quad \rho=\rho_{+}-\rho_{-},
$$

необходимо и достаточно выполнение следующих двух условий:

1) $\quad n V^{(n)}(t b(n)) \longrightarrow \rho_{+} t^{-\alpha}, \quad n W^{(n)}(t b(n)) \longrightarrow \rho_{-} t^{-\alpha}$,

2) $\quad \lim _{\varepsilon \rightarrow 0} \limsup _{n \rightarrow \infty} n \int_{|t|<\varepsilon} t^{2} F^{(n)}(b(n) d t)=0$.

Для доказательства теоремы 1 нам достаточно убедиться, что условия [UR] влекут за собой (4), (5). Выполнение (4) почти очевидно: для каждого фиксированного $t$ при $n \rightarrow \infty$

$$
n V^{(n)}(t b(n)) \sim n \rho_{+} G(t b(n)) \sim n \rho_{+} t^{-\alpha} G(b(n)) \sim \rho_{+} t^{-\alpha}
$$

(если $\rho_{+}=0$, то $n V^{(n)}(t b(n)) \rightarrow 0$ при $n \rightarrow \infty$ ).

Аналогичное соотношение справедливо для $W^{(n)}$.

Для проверки (5) рассмотрим сначала

$$
I_{+}=n \int_{0}^{\varepsilon} t^{2} F(b(n) d t)=-\frac{n}{b^{2}(n)} \int_{0}^{\varepsilon b(n)} u^{2} d V(u) \leqslant \frac{2 n}{b^{2}(n)} \int_{0}^{\varepsilon b(n)} u V(u) d u .
$$

Пусть $t_{1}, n_{1}$ таковы, что в силу условий [UR] $V(u)<2 \rho_{+} G(u)$ при $u \geqslant t_{1}$, $n \geqslant n_{1}$. Тогда при $n \geqslant n_{1}$

$$
I_{+} \leqslant \frac{n t_{1}^{2}}{b^{2}(n)}+\frac{4 n \rho_{+}}{b^{2}(n)} \int_{t_{1}}^{\varepsilon b(n)} u G(u) d u,
$$

где последнее слагаемое в правой части в силу свойств правильно меняющихся функций асимптотически эквивалентно при $n \rightarrow \infty(b(n) \rightarrow \infty)$ величине

$$
\frac{4 n \rho_{+}(\varepsilon b(n))^{2} G(\varepsilon b(n))}{b^{2}(n)(2-\alpha)} \sim \frac{4 \varepsilon^{2-\alpha} \rho_{+}}{2-\alpha} n G(b(n)) \sim \frac{4 \varepsilon^{2-\alpha} \rho_{+}}{2-\alpha} .
$$

Первое слагаемое в правой части (6) сходится к нулю при $n \rightarrow \infty$, так как $b(n)=n^{1 / \alpha} L_{b}(n)$ ( $L_{b}-$ медленно меняюшаяся функция), $\alpha<2$, $b^{2}(n) \gg n$.

Из сказанного следует, что

$$
\lim _{\varepsilon \rightarrow 0} \limsup _{n \rightarrow \infty} I_{+}=0 .
$$

Аналогично определяется и оценивается значение $I_{-}$. Выполнение условий (4), (5), а вместе с ними и утверждение теоремы, доказаны. 
Обозначим далее через $X^{(\alpha, \rho)}(t)$ устойчивый случайный процесс на $[0, T], T>0$, с распределением $\mathbf{F}^{(\alpha, \rho)}$ для $X^{(\alpha, \rho)}(1)$ и положим

$$
X_{n}(t)=\frac{S_{[n t]}}{b(n)}, \quad t \in[0, T] .
$$

Пусть $D(0, T)$ - пространство функций на $[0, T]$ без разрывов второго рода с метрикой Скорохода

$$
d\left(f_{1}, f_{2}\right)=\inf _{\lambda} \sup _{t}\left[\left|f_{2}(t)-f_{1}(\lambda(t))\right|+|\lambda(t)-t|\right]
$$

где инфимум берется по всем непрерывным возрастающим функциям $\lambda(t)$ на $[0, T]$ таким, что $\lambda(0)=0, \lambda(T)=1$.

Нам понадобятся также условия слабой сходимости при $n \rightarrow \infty$ распределений процессов $X_{n}(\cdot)$ в пространстве $D(0, T)$ к распределению процесса $X^{(\alpha, \rho)}(\cdot)$.

Рассмотрим произвольное фиксированное $\Delta \in(0, T)$ и совокупность случайных величин $\xi_{k+1}, \ldots, \xi_{k+[n \Delta]}$ из $n$-й серии. Для них введем усредненное распределение

$$
\mathbf{F}_{(k, \Delta)}=\frac{1}{[n \Delta]} \sum_{j=k+1}^{k+[n \Delta]} \mathbf{F}_{j}
$$

и усредненные хвосты

$$
F_{(k, \Delta)}(t)=\frac{1}{[n \Delta]} \sum_{j=k+1}^{k+[n \Delta]} \mathbf{F}_{j}(t), \quad V_{(k, \Delta)}(t)=\frac{1}{[n \Delta]} \sum_{j=k+1}^{k+[n \Delta]} V_{j}(t)
$$

Для сходимости процессов $X_{n}(\cdot)$ к однородным устойчивым процессам нам понадобятся однородные условия равномерного правильного изменения, в основе которых лежат условия [UR].

$[\mathrm{UR}]_{\Delta}$. Существует Фиксированная правильно меняющаяся Функиия $G(t)=t^{-\alpha} L(t)$ такая, что при каждом фиксированном $\Delta>0$

$$
\begin{aligned}
\lim _{t \rightarrow \infty, n \rightarrow \infty} \frac{n F_{(k, \Delta)}(t)}{G(t)} & =1, \\
\lim _{t \rightarrow \infty, n \rightarrow \infty} \frac{n V_{(k, \Delta)}(t)}{G(t)} & =\rho_{+},
\end{aligned}
$$

где сходимость к пределам равномерна по $k \leqslant n(T-\Delta)$.

Теорема 2. Пусть усредненные распределения $\mathbf{F}_{(k, \Delta)}$ удовлетворяют условиям $[\mathrm{UR}]_{\Delta}$. Тогда при $n \rightarrow \infty$

$$
X_{n}(\cdot) \Rightarrow X^{(\alpha, \rho)}(\cdot)
$$

т.е. распределения прочесса $X_{n}(\cdot)$ слабо сходятся в $D(0, T) \kappa$ распределению прочесса $X^{\alpha, \rho}(\cdot)$. 
Очевидно, что условия теоремы 2 будут выполнены, если найдется $n_{0}=o(n), n_{0} \rightarrow \infty$ при $n \rightarrow \infty$, такое, что усредненные в $n$-й серии распределения

$$
\mathbf{F}_{(k)}=\frac{1}{n_{0}} \sum_{j=k+1}^{k+n_{0}} \mathbf{F}_{j}
$$

равномерно по $k$ удовлетворяют условиям [UR] (при выполнении $\left.\mathbf{E} \xi_{j}=0\right)$.

Доказательств о те о е мы 2. Положим, не ограничивая общности, $T=1$. Для того чтобы установить сходимость (8), мы должны убедиться, что выполнены следуюшие два условия (см., например, $[8, \S 15])$.

1. Конечномерные распределения $X_{n}(\cdot)$ сходятся к соответствующим распределениям $X^{(\alpha, \rho)}(\cdot)$.

2. Выполнены условия компактности (плотности) семейства распределений процессов $X_{n}(\cdot)$ в пространстве $D(0,1)$.

Сходимость конечномерных распределений вытекает из теоремы 1 , так как усредненные распределения $\mathbf{F}_{(k, \Delta)}$ удовлетворяют условиям [UR]. Кроме того, условия $[\mathrm{UR}]_{\Delta}$ влекут за собой выполнение условий пренебрежимой малости [S]. Действительно, при $j \in(k, k+\Delta n)$ и всех достаточно больших $n$

$$
\mathbf{P}\left\{\left|\xi_{j}\right|>\varepsilon b(n)\right\} \leqslant n \Delta F_{(k, \Delta)}(\varepsilon b(n)) \leqslant 2 \Delta n G(\varepsilon b(n)) \leqslant 3 \Delta \varepsilon^{-\alpha} .
$$

Так как $\Delta$ может быть выбрано сколь угодно малым, а левая часть этих неравенств от $\Delta$ не зависит, то с необходимостью получаем

$$
\max _{j \leqslant n} \mathbf{P}\left\{\left|\xi_{j}\right|>\varepsilon b(n)\right\} \longrightarrow 0 \quad \text { при } \quad n \rightarrow \infty .
$$

Для выполнения условий компактности достаточно, чтобы при каждых фиксированных $t_{1}<t<t_{2}$ выполнялось

$$
\limsup _{n \rightarrow \infty} \mathbf{P}\left\{\left|X_{n}(t)-X_{n}\left(t_{1}\right)\right| \geqslant v,\left|X_{n}\left(t_{2}\right)-X_{n}(t)\right| \geqslant v\right\} \leqslant c v^{-\gamma}\left|t_{2}-t_{1}\right|^{1+\delta}
$$

при некоторых $c<\infty, \gamma>0, \delta>0$ (см. [9]). В силу сходимости конечномерных распределений при $n \rightarrow \infty, \Delta=t-u>0, m=[n t]-[n u] \sim n \Delta$, $S_{m}^{\prime}=S_{[n t]}-S_{[n u]}$ выполняется

$$
\begin{aligned}
\mathbf{P}\left\{\left|X_{n}(t)-X_{n}(u)\right| \geqslant v\right\} & =\mathbf{P}\left\{\left|S_{m}^{\prime}\right| \geqslant v b(n)\right\} \sim \mathbf{P}\left\{\left|X^{(\alpha, \rho)}\right| \geqslant \frac{v b(n)}{b(m)}\right\} \\
& \sim \mathbf{P}\left\{\left|X^{(\alpha, \rho)}\right|>v \Delta^{-1 / \alpha}\right\} \leqslant c v^{-\alpha} \Delta .
\end{aligned}
$$

Последнее неравенство вытекает из свойств устойчивых распределений.

Так как события под знаком вероятности в (9) независимы, то в силу (10) эта вероятность не превосходит $c^{2} v^{-2 \alpha}\left(t_{2}-t_{1}\right)^{2}$. Неравенство (9) выполнено. Теорема 2 доказана. 
3. Переходные явления. Вернемся теперь к исходной постановке задачи о предельном распределении максимума сумм

$$
\bar{Z}_{n}=\max _{k \leqslant n} Z_{k}
$$

нечентрированных слагаемых $\zeta_{i}$. Теперь в качестве параметра схемы серий мы будем рассматривать значения $a \rightarrow 0$, а под $n$ понимать некоторую функцию $n=n(a) \uparrow \infty$ при $a \downarrow 0$. Основные предпосылки для изучения распределения $\bar{Z}_{n}$, связанные со свойствами иентрированных слагаемых $\xi_{i}$, мы изложили в п. 2. Как уже отмечалось, другой предпосылкой является правильное изменение средних $a_{i}=-\mathbf{E} \zeta_{i}$. Мы будем предполагать в дальнейшем, что выполнено следуюшее условие.

[g]. Параметр схемы серий а сходится $\kappa$, а средние значения $a_{i}$ таковы, что существует

$$
\lim _{a \rightarrow 0, k \rightarrow \infty} \frac{g_{k}}{a k}=1, \quad g_{k}=\sum_{i=1}^{k} a_{i}, \quad \max _{k \leqslant 1 / a}\left|a_{k}\right|<c .
$$

Другими словами, средние значения $g_{k}=-\mathbf{E} Z_{k}$ ведут себя асимптотически линейно с малым коэффициентом роста $a$.

В новых условиях через $\bar{S}_{n}(a)$ мы обозначим функционал

$$
\bar{S}_{n}(a)=\max _{k \leqslant n}\left(S_{k}-g_{k}\right),
$$

так что $\bar{S}_{n}(a)=\bar{Z}_{n}$.

Применительно к приложениям условие [g] можно назвать условием асимптотически равномерной тяжелой нагрузки. Можно рассматривать и асимптотически нелинейные «границы» $g_{k}$, как это сделано при изучении вероятностей больших уклонений $\bar{S}_{n}(a)$ в [9]. В этом случае, предполагая известную правильность изменения $g_{k}$, также можно исследовать предельное распределение $\bar{S}_{n}(a)$, но оно будет иным, чем в теореме 3 , приведенной ниже.

Мы можем сформулировать теперь основные утверждения настояшей работы. Положим

$$
D(t)=t G(t), \quad d(v)=D^{(-1)}(v)=\inf \{u: D(u)<v\}, \quad n_{1}=n_{1}(a)=\frac{d(a)}{a} .
$$

Нетрудно проверить, что если $G$ - правильно меняющаяся функция с показателем $-\alpha$, то $d$ и $n_{1}$ также будут правильно меняющимися функциями с показателями $1 /(1-\alpha)$ и $\alpha /(1-\alpha)$ соответственно.

Теорема 3. Пусть выполнень условия теоремь 2 при замене в них $n$ на $n_{1}=d(a) / a$ (т.е. выполнены условия $[\mathrm{UR}]_{\Delta}$ при замене в них $n$ на $\left.n_{1}\right)$. Пусть далее вьполнено условие $[g], a \rightarrow 0 u n=n(a)$ таково, 
что существует

$$
\lim _{a \rightarrow 0} \frac{n(a)}{n_{1}(a)}=T<\infty
$$

Tогдa

$$
\frac{\bar{S}_{n}(a)}{d(a)} \Rightarrow Z^{(\alpha, \rho)}(T)
$$

m.е. распределение $\bar{S}_{n}(a) / d(a)$ слабо сходится при а $\rightarrow 0 \kappa$ распределению

$$
Z^{(\alpha, \rho)}(T)=\sup _{u \leqslant T}\left(X^{(\alpha, \rho)}(u)-u\right)
$$

Можно показать, что распределение случайной величины $Z^{(\alpha, \rho)}(T)$ непрерывно. Это вместе с теоремой 3 будет означать, что при всех $v$ и при $a \rightarrow 0$

$$
\mathbf{P}\left\{\frac{\bar{S}_{n}(a)}{d(a)}>v\right\} \rightarrow \mathbf{P}\left\{Z^{(\alpha, \rho)}(T)>v\right\} .
$$

Доказательство те оремы 3. Пусть, как и прежде, $b(n)=G^{(-1)}(1 / n)$. Сначала будем считать для простоты, что $a_{i}=a$ и функция $G(t)$ непрерывна при достаточно больших $t$. Заметим предварительно, что тогда

$$
d(a)=b\left(n_{1}\right)
$$

Это следует из равенств

$$
\begin{gathered}
a \equiv D(d(a))=d(a) G(d(a)), \quad G(d(a))=\frac{a}{d(a)}=\frac{1}{n_{1}} \\
d(a)=G^{-1}\left(\frac{1}{n_{1}}\right)=b\left(n_{1}\right) .
\end{gathered}
$$

Поэтому

$$
\frac{\bar{S}_{n}(a)}{d(a)}=\max _{k \leqslant n}\left(\frac{S_{k}}{b\left(n_{1}\right)}-\frac{a k}{b\left(n_{1}\right)}\right)=\max _{k \leqslant n}\left(X_{n_{1}}\left(\frac{k}{n_{1}}\right)-\frac{k \theta}{n_{1}}\right),
$$

где в силу (13)

$$
\theta=\frac{n_{1} a}{b\left(n_{1}\right)}=\frac{d(a)}{b\left(n_{1}\right)}=1
$$

Но функционал

$$
f_{T}(X)=\sup _{u \leqslant T}(X(u)-u)
$$

непрерывен в метрике Скорохода и обладает свойством

$$
f_{T}\left(X_{n_{1}}\right)=\max _{k \leqslant n}\left(X_{n_{1}}\left(\frac{k}{n_{1}}\right)-\frac{k}{n_{1}}\right),
$$


так что в силу (14)

$$
\frac{\bar{S}_{n}(a)}{d(a)}=f_{T}\left(X_{n_{1}}\right) .
$$

Остается воспользоваться теоремой 2 .

В общем случае, когда функция $G(t)$ не является непрерывной при больших $t$, вместо равенства (13) будет иметь место асимптотическая эквивалентность $d(a) \sim b\left(n_{1}\right)$ при $a \rightarrow 0$ и все рассуждения доказательства (при замене в них ряда равенств на асимптотическую эквивалентность) сохранятся. То же самое произойдет, если $a k$ в (14) заменить в соответствии с условием $[g]$ на $g_{k} \sim a k$ при $a \rightarrow 0, k \rightarrow \infty$. При этом вторая часть условия $[g]$ обеспечивает отсутствие «выбросов» у нормированной границы вблизи начала координат.

Теорема 3 доказана.

Рассмотрим теперь случай, когда $n \gg n_{1}$ и, в частности, случай $n=\infty$.

Положим $Z^{(\alpha, \rho)}(\infty)=Z^{(\alpha, \rho)}$ :

Теорема 4. Пусть выполнены условия теоремы 3 и $T=\infty$. Тогда nрu $a \rightarrow 0$

$$
\frac{\bar{S}_{\infty}(a)}{d(a)} \Rightarrow Z^{(\alpha, \rho)} .
$$

Доказательств о. Пусть

$$
\eta(x, a)=\min \left\{k: S_{k}-g_{k}>x\right\} .
$$

Положим для краткости

$$
X(n, a)=\frac{\bar{S}_{n}(a)}{d(a)} .
$$

Выберем большое фиксированное число $T$ и обозначим $n_{T}=n_{1} T$. Тогда

$$
\mathbf{P}\{X(\infty, a)>v\}=\mathbf{P}\left\{X\left(n_{T}, a\right)>v\right\}+\mathbf{P}\left\{\infty>\eta(v d(a), a)>n_{T}\right\} .
$$

По теореме 3 первое слагаемое в правой части (16) сходится при $a \rightarrow 0$ к $\mathbf{P}\left\{Z^{(\alpha, \rho)}(T)>v\right\}$. Для второго слагаемого в силу следствий $3.1,3.2$ в [9] при $x=v d(a)$ получаем

$$
\begin{aligned}
\mathbf{P}\left\{\infty>\eta(v d(a), a)>n_{T}\right\} & =\mathbf{P}\left\{\infty>\eta(x, a)>\frac{T x}{a v}\right\} \leqslant c n_{1} \widehat{V}_{(1)}(x)\left\{\frac{T}{v}\right\}^{1-\alpha} \\
& \leqslant c_{1} n_{1} F_{(1)}(x)\left\{\frac{T}{v}\right\}^{1-\alpha}
\end{aligned}
$$

где хвосты $\widehat{V}_{(1)}=\max \left(V_{(1)}, W_{(1)}\right), F_{(1)}$ соответствуют усредненному распределению

$$
\mathbf{F}_{(1)}=\frac{1}{n_{1}} \sum_{j=1}^{n_{1}} \mathbf{F}_{j}
$$


(можно рассматривать также усредненные распределения $\mathbf{F}_{(T)}=$ $\left.n_{T}^{-1} \sum_{j=1}^{n_{T}} \mathbf{F}_{j}\right)$. Заметим теперь, что в (17)

$$
n_{1} F_{(1)}(x)=\frac{d(a)}{a} F_{(1)}(v d(a)) \sim \frac{d(a)}{a} G(v d(a)) \sim \frac{v^{-\alpha}}{a} D(d(a)) \sim v^{-\alpha}
$$

и, стало быть, равномерно по $a$

$$
R(v, T) \equiv \mathbf{P}\left\{\infty>\eta(v d(a), a)>n_{T}\right\} \leqslant \frac{c_{1}}{v} T^{1-\alpha},
$$

где правая часть выбором $T$ может быть сделана сколь угодно малой. Из сказанного следует, что

$$
\begin{aligned}
\varlimsup_{a \rightarrow 0} \mathbf{P}\{X(\infty, a)>v\} & \leqslant \mathbf{P}\left\{Z^{(\alpha, \rho)}(T)>v\right\}+R(v, T) \\
& \leqslant \mathbf{P}\left\{Z^{(\alpha, \rho)}>v\right\}+R(v, T) .
\end{aligned}
$$

Так как левая часть этого неравенства от $T$ не зависит, а правая может быть выбором $T$ сделана сколь угодно близкой к $\mathbf{P}\left\{Z^{(\alpha, \rho)}>v\right\}$, то

$$
\varlimsup_{a \rightarrow 0} \mathbf{P}\{X(\infty, a)>v\} \leqslant \mathbf{P}\left\{Z^{(\alpha, \rho)}>v\right\} .
$$

С другой стороны, при любом $T$

$$
\varliminf_{a \rightarrow 0} \mathbf{P}\{X(\infty, a)>v\} \geqslant \mathbf{P}\left\{Z^{(\alpha, \rho)}(T)>v\right\},
$$

где $\mathbf{P}\left\{Z^{(\alpha, \rho)}(T)>v\right\} \uparrow \mathbf{P}\left\{Z^{(\alpha, \rho)}>v\right\}$ при $T \uparrow \infty$. Учитывая, как и в (18), независимость левой части от $T$, мы получаем

$$
\varliminf_{a \rightarrow 0} \mathbf{P}\{X(\infty, a)>v\} \geqslant \mathbf{P}\left\{Z^{(\alpha, \rho)}>v\right\}
$$

Сказанное означает, что существует

$$
\lim _{a \rightarrow \infty} \mathbf{P}\{X(\infty, a)>v\}=\mathbf{P}\left\{Z^{(\alpha, \rho)}>v\right\} .
$$

Теорема доказана.

4. О явном виде предельных распределений. Найти в общем случае явный вид собственных предельных распределений в теоремах 3,4 (т.е. распределений $Z^{(\alpha, \rho)}(T)$ ) вряд ли возможно (вероятности больших уклонений величин $\bar{S}_{n}(a) / d(a)$ изучены в теореме 3.1 и следствии 3.1 в [9]). Однако в двух крайних случаях $\rho=-1$ и $\rho=1$ при $T=\infty$ это удается сделать. Рассмотрим сначала случай $\rho=-1$ или, что то же, $V(t)=o(W(t))$ при $t \rightarrow \infty$. Тогда, очевидно, $W(t) \sim G(t)$.

Теорема 5. Справедливо равенство

$$
\mathbf{P}\left\{Z^{(\alpha,-1)}>v\right\}=e^{-b v},
$$

аде $b=(\Gamma(2-\alpha) /(\alpha-1))^{1 /(1-\alpha)}$. 
Если принять $\Gamma(1-\alpha)=\Gamma(2-\alpha) /(1-\alpha)$ при $\alpha \in(1,2)$, то $b$ можно записать также в виде $b=(-\Gamma(1-\alpha))^{1 /(1-\alpha)}$.

Д о к а з а т е л ь с т в о. Из определения случайных величин $Z^{(\alpha, \rho)}$ видно, что оно не зависит от вида хвостов $V$ и $W$ и определяется лишь параметрами $\alpha$ и $\rho$. Поэтому, чтобы найти распределение $Z^{(\alpha,-1)}$, достаточно найти предельное распределение $\bar{S}_{\infty}(a) / d(a)$ при $a \rightarrow 0$ и какихнибудь конкретных $V$ и $W$, удовлетворяющих условиям теорем 3,4 . Возьмем, например, в качестве исходной последовательности $\xi_{1}, \xi_{2}, \ldots$ случайные величины, распределение $\mathbf{F}$ которых имеет хвосты

$$
V(t)=\frac{1}{2} e^{-\gamma t}, \quad W(t)=\frac{1}{2} \min (1, t)^{-\alpha}, \quad t>0,
$$

при подходящем $\gamma>0$ таком, что $\mathbf{E} \xi_{j}=0$. Так как в нашем случае $a_{+}=\mathbf{E} \max \left(0, \xi_{j}\right)=1 /(2 \gamma), a_{-}=\mathbf{E} \min \left(0, \xi_{j}\right)=-\frac{1}{2}(1+1 /(\alpha-1))=$ $-\alpha /(2(\alpha-1))$, то при $\gamma=(\alpha-1) / \alpha$ будет выполняться $\mathbf{E} \xi_{j}=a_{+}+a_{-}=0$. Далее, $V(t)=o(W(t))$ и выполнены все условия теорем 3,4 при $\rho=-1$, $d(a) \sim(2 a)^{1 /(1-\alpha)}$.

Но для экспоненциальных правых хвостов распределение $\bar{S}(a)$ известно в явном виде. Именно, в этом случае (см., например, [3])

$$
\mathbf{E} e^{\lambda \bar{S}(a)}=p+\frac{(1-p) \lambda_{1}}{\lambda_{1}-\lambda}, \quad p=\mathbf{P}\{\bar{S}(a)=0\}
$$

так что хвост распределения $\bar{S}(a)$ также является экспоненциальным:

$$
\mathbf{P}\{\bar{S}(a)>x\}=(1-p) e^{-\lambda_{1} x}, \quad x>0 .
$$

Здесь $\lambda_{1}>0$ - решение уравнения $\psi(\lambda)=1, \psi(\lambda)=\mathbf{E} e^{\lambda(\xi-a)}$ (ясно, что $p \rightarrow 0$ при $a \rightarrow 0)$. Для отыскания значения $\lambda_{1}$ надо найти асимптотическое представление для

$$
\psi(\lambda)=e^{-a \lambda} \int_{-\infty}^{\infty} e^{\lambda t} \mathbf{F}(d t)
$$

при $\lambda \rightarrow 0$.

Лемма 1. Справедливо следуюшее соотношение.

$$
\psi(\lambda)=1-\lambda a+\frac{W(1 / \lambda)}{(\alpha-1)} \Gamma(2-\alpha)(1+o(1))
$$

$n p u \lambda \rightarrow 0$,

$$
\lambda_{1}^{-1} \sim D^{(-1)}\left(\frac{a}{b_{1}}\right) \sim b d(a), \quad b_{1}=b^{1-\alpha}=\frac{\Gamma(2-\alpha)}{\alpha-1} .
$$

Д о к а з а т е л с т в о. Имеем $e^{a \lambda} \psi(\lambda)=I_{1}+I_{2}$, где

$$
I_{1}=\int_{-\infty}^{0} e^{\lambda t} \mathbf{F}(d t)=W(0)+\lambda \int_{-\infty}^{0} t \mathbf{F}(d t)+\int_{-\infty}^{0}\left(e^{\lambda t}-1-\lambda t\right) \mathbf{F}(d t)
$$


Здесь

$$
\int_{-\infty}^{0}\left(e^{\lambda t}-1-\lambda t\right) \mathbf{F}(d t)=\lambda \int_{0}^{\infty}\left(1-e^{-\lambda t}\right) W(t) d t=\lambda^{2} \int_{0}^{\infty} e^{-\lambda t} W^{I}(t) d t,
$$

где $W^{I}(t)=\int_{t}^{\infty} W(u) d u=t W(t) /(\alpha-1)$ при $t \geqslant 1$. Поэтому при $\lambda \rightarrow 0$ $(\lambda t=u)$

$$
\lambda^{2} \int_{0}^{\infty} e^{-\lambda t} W^{I}(t) d t \sim \lambda W^{I}\left(\frac{1}{\lambda}\right) \int_{0}^{\infty} e^{-u} u^{-\alpha+1} d u \sim \frac{W(1 / \lambda)}{\alpha-1} \Gamma(2-\alpha) .
$$

Таким образом,

$$
I_{1}=\frac{1}{2}+\lambda a_{-}+\frac{W(1 / \lambda)}{\alpha-1} \Gamma(2-\alpha)(1+o(1)) .
$$

Очевидно также, что для $\mathbf{E}\left(\xi^{+}\right)^{2}<\infty$

$$
I_{2}=\frac{1}{2}+\lambda a_{+}+O\left(\lambda^{2}\right), \quad e^{-a \lambda}=1-a \lambda+O\left(\lambda^{2}\right) .
$$

Это доказывает (22).

Далее, при $b_{1}=\Gamma(2-\alpha) /(\alpha-1)$ искомое значение $\lambda_{1}$ есть решение уравнения

или, что то же,

$$
\lambda a=b_{1} W\left(\frac{1}{\lambda}\right)(1+o(1))
$$

$$
\lambda a=b_{1} G\left(\frac{1}{\lambda}\right)(1+o(1)), \quad D\left(\frac{1}{\lambda}\right) \sim \frac{a}{b_{1}},
$$

поскольку в нашем случае $W(t) \sim G(t)$ при $t \rightarrow \infty$. Отсюда находим

$$
\lambda_{1}^{-1} \sim d\left(\frac{a}{b_{1}}\right) \sim b_{1}^{1 /(\alpha-1)} d(a) .
$$

Лемма 1 доказана.

Возврашаясь к (21) и полагая $x=v d(a)$, находим

$$
\mathbf{P}\left\{\frac{\bar{S}(a)}{d(a)}>v\right\} \sim e^{-b v}, \quad \mathbf{P}\left\{Z^{(\alpha,-1)}>v\right\}=e^{-b v} .
$$

Теорема 5 доказана.

Рассмотрим теперь вторую возможность $\rho=1$ отыскания явного вида распределения $Z^{(\alpha, \rho)}$, т.е. случай $W(t)=o(V(t))$ при $t \rightarrow \infty$. На этот раз удается найти в явном виде лишь характеристическую функцию $Z^{(\alpha, 1)}$.

Теорема 6. Справедливо следующее равенство:

$$
\mathbf{E} e^{i \lambda Z^{(\alpha, 1)}}=\left[1+\frac{i \phi|\lambda|^{\alpha-1}}{\alpha-1} A(\alpha-1, \phi)\right]^{-1}, \quad \phi=\operatorname{sign} \lambda,
$$

где $A(\gamma, \phi)=\int_{0}^{\infty} e^{i v \phi} v^{-\gamma} d v=\Gamma(1-\gamma) e^{i \phi(1-\gamma) \pi / 2}$. 
Нетрудно заметить следующую связь между характеристическими функциями случайных величин $Z^{(\alpha, 1)}$ и $X^{(\alpha-1,1)}$ :

$$
\mathbf{E} e^{i \mu Z^{(\alpha, 1)}}=\left[1-\ln \varphi^{(\alpha-1,1)}(\mu)\right]^{-1} .
$$

Вероятности больших уклонений для $Z^{(\alpha, \rho)}$ (или $\left.\bar{S}(a) / d(a)\right)$ изучены в [9].

Д ок а з тель с т о т е о ремы 6. Используя те же рассуждения, что и в теореме 5 , мы можем считать, не ограничивая общности (при отыскании распределения $Z^{(\alpha, 1)}$ ), что $\xi_{j}$ одинаково распределены и имеют хвосты того же вида, что и в (19), но $V$ и $W$ надо поменять местами, так что

$$
W(t)=\mathbf{P}\left\{\xi_{j}<-t\right\}=\frac{1}{2} e^{-\gamma t}, \quad \gamma=-\frac{1}{2} a_{-}^{-1}, \quad a_{-}=\mathbf{E} \min (0, \xi) .
$$

Тогда по-прежнему $\mathbf{E} \xi_{j}=0$, а первая отрицательная сумма $\chi_{-}$будет иметь экспоненциальное распределение: $\mathbf{P}\left\{\chi_{-}<-t\right\}=e^{-\gamma t}, \mathbf{E} e^{i \lambda \chi-}=$ $\gamma /(i \lambda+\gamma)$. В силу факторизационных тождеств (см., например, [3])

$$
\mathbf{E} e^{i \lambda \bar{S}(a)}=\frac{p}{1-\varphi_{a}(\lambda)}\left[1-\mathbf{E} e^{i \lambda \chi_{-}}\right]=\frac{p i \lambda}{\left(1-\varphi_{a}(\lambda)\right)(i \lambda+\gamma)},
$$

где

$$
\varphi_{a}(\lambda)=\mathbf{E} e^{i \lambda(\xi-a)}, \quad p=\mathbf{P}\{\bar{S}(a)=0\} .
$$

Выясним асимптотическое поведение $\varphi_{a}(\lambda)-1$ при $\lambda \rightarrow 0$. Для $\varphi(\lambda)=\varphi_{0}(\lambda)=\mathbf{E} e^{i \lambda \xi}$ имеем

$$
\varphi(\lambda)-1=\phi \int_{0}^{|\lambda|} \varphi^{\prime}(u \phi) d u, \quad \phi=\operatorname{sign} \lambda
$$

где

$$
\varphi^{\prime}(\lambda)=i\left[-\int_{0}^{\infty} x e^{i \lambda x} d V(x)+\int_{0}^{\infty} x e^{-i \lambda x} d W(x)\right] \equiv I_{+}(\lambda)+I_{-}(\lambda) .
$$

Так как $x d V(x)=d(x V(x))-V(x) d x$, то

$$
\begin{aligned}
I_{+}(\lambda) \equiv & -i \int_{0}^{\infty} x e^{i \lambda x} d V(x)=-\lambda \int_{0}^{\infty} x V(x) e^{i \lambda x} d x+i V^{I}(0) \\
& -\lambda \int_{0}^{\infty} V^{I}(x) e^{i \lambda x} d x=i V^{I}(0)-\lambda \int_{0}^{\infty} \tilde{V}(x) e^{i \lambda x} d x
\end{aligned}
$$

где

$$
V^{I}(x)=\int_{x}^{\infty} V(u) d u, \quad \tilde{V}(x)=x V(x)+V^{I}(x) .
$$

Здесь в силу наших предположений

$$
V^{I}(x) \sim \frac{x V(x)}{\alpha-1}, \quad \tilde{V}(x) \sim \frac{\alpha x V(x)}{\alpha-1}
$$


при $x \rightarrow \infty$. Далее, при $m=1 /|\lambda| \rightarrow \infty, z=x / m$ находим

$$
\begin{aligned}
& \int_{0}^{\infty} \tilde{V}(x) e^{i \lambda x} d x=\int_{0}^{1}+\int_{1}^{\infty} ; \\
m^{-1} \int_{1}^{\infty} & =\int_{m^{-1}}^{\infty} \tilde{V}(z m) e^{i z \phi} d z \sim \tilde{V}(m) \int_{0}^{\infty} z^{-\alpha+1} e^{i z \phi} d z \\
& \sim \frac{\alpha m V(m)}{\alpha-1} A(\alpha-1, \phi),
\end{aligned}
$$

где

$$
A(\gamma, \phi)=\int_{0}^{\infty} e^{i v \phi} v^{-\gamma} d v=\Gamma(1-\gamma) e^{i \phi(1-\gamma) \pi / 2}
$$

(см. [10]). Так как $m^{-1} \int_{0}^{1} \tilde{V}(x) e^{i \lambda x} d x=O\left(m^{-1}\right)$, то в итоге получаем, что при $\lambda \rightarrow 0$

$$
I_{+}(\lambda)=i V^{I}(0)-\frac{\alpha V(m)}{\lambda(\alpha-1)} A(\alpha-1, \phi)(1+o(1)) .
$$

Аналогично находим

$$
I_{-}(\lambda)=-i W^{I}(0)+O\left(m^{-1}\right)
$$

Таким образом,

$$
I_{+}(\lambda)+I_{-}(\lambda)=-\frac{\alpha V(m)}{\lambda(\alpha-1)} A(\alpha-1, \phi)(1+o(1)) .
$$

Так как при $\lambda \rightarrow 0$

$$
\int_{0}^{|\lambda|} \frac{1}{u} V\left(\frac{1}{u}\right) d u \sim \frac{1}{\alpha} V\left(\frac{1}{|\lambda|}\right)
$$

то, интегрируя $I_{+}(u)+I_{-}(u)$ в пределах от 0 до $|\lambda|$, мы в силу $(24),(25)$ находим

$$
\varphi(\lambda)-1=-\frac{V(m)}{\alpha-1} A(\alpha-1, \phi)(1+o(1)) .
$$

Стало быть,

$$
\begin{aligned}
1-\varphi_{a}(\lambda) & =i \lambda a+\frac{V(m)}{\alpha-1} A(\alpha-1, \phi)(1+o(1)) \\
& =i \lambda a\left(1-\frac{i V(m)}{\lambda a(\alpha-1)} A(\alpha-1, \phi)(1+o(1))\right) \\
\mathbf{E} e^{i \lambda \bar{S}(a)} & =\frac{p}{a}\left[1+\frac{i V(m)}{\lambda a(\alpha-1)} A(\alpha-1, \phi)(1+o(1))\right]^{-1}(i \lambda+\gamma)^{-1}
\end{aligned}
$$

Полагая $\lambda=\mu / d(a)$ при фиксированном $\mu$ и $a \rightarrow 0$, находим

$\mathbf{E} e^{i \mu \bar{S}(a) / d(a)}=\frac{p}{a q}\left[1+\frac{i \phi D(d(a) /|\mu|)}{a(\alpha-1)} A(\alpha-1, \phi)(1+o(1))\right]^{-1}\left(1+\frac{i \mu}{d(a)}\right)^{-1}$, 
где $a^{-1} D(d(a) /|\mu|) \sim|\mu|^{\alpha-1}, \phi=\operatorname{sign} \mu$. Так как

$$
\frac{\bar{S}(a)}{d(a)} \rightarrow Z^{(\alpha, 1)}
$$

то отсюда следует, что с необходимостью $p /(a q) \rightarrow 1$,

$$
\mathbf{E} e^{i \mu \bar{S}(a) / d(a)} \longrightarrow\left[1+\frac{i \phi|\mu|^{\alpha-1}}{\alpha-1} A(\alpha-1, \phi)\right]^{-1} .
$$

Теорема 6 доказана.

Так как $\rho=-1$ тогда и только тогда, когда процесс $X^{(\alpha, \rho)}(t)$ (или $\left.Y(t)=X^{(\alpha, \rho)}-t\right)$ не содержит положительных скачков (непрерывен сверху), то экспоненциальный характер распределения $Z^{(\alpha,-1)}$ будет вытекать (вместе с обратным утверждением) из следующего просто доказываемого общего факта.

Теорема 7. Пусть $Y(t), t \geqslant 0,-$ произвольньий однородньгй процесс с независимьми приращениями такой, что $Z=\sup _{t \geqslant 0} Y(t)<$ $\infty$ п.н. Для того чтобы случайная величина $Z$ имела экспоненииальное распределение

$$
\mathbf{P}\{Z>x\}=e^{-b x}, \quad b>0,
$$

необходимо и достаточно, чтобы траектории $Y(t)$ были непрерьвнь сверху (не имели положительных скачков).

Доказ ат ельст в о. Положим $\eta(x)=\inf \{t \geqslant 0: Y(t)>x\}$, если $\bar{Z}>x$, и $\eta(x)=\infty$, если $\bar{Z} \leqslant x$. На множестве $\bar{Z}>x$ определена случайная величина $\chi(x)=Y(\eta(x))-x$. Пусть для определенности траектории процесса $Y(t)$ непрерывны справа. Тогда $\chi(x) \geqslant 0 ;$ при этом $\chi(x) \equiv 0$ тогда и только тогда, когда процесс $Y(t)$ непрерывен сверху.

Для произвольного $y, 0<y<x$, имеем

$$
P(x) \equiv \mathbf{P}\{Z>x\}=\mathbf{E}[\mathbf{P}\{Z>x \mid \eta(y)\} ; \eta(y)<\infty],
$$

где в силу строго марковского свойства $Y(\cdot)$

$$
\mathbf{P}\{Z>x \mid \eta(y)\}=\mathbf{P}\left\{Z^{\prime}>x-y-\chi(y)\right\} .
$$

Здесь $Z^{\prime}=\sup _{t \geqslant 0} Y^{\prime}(t)$, процесс $Y^{\prime}(t)$ устроен так же, как $Y(t)$, и не зависи г от $Y(t)$. Поэтому, если процесс $Y(\cdot)$ непрерывен сверху, то $\mathbf{P}\{Z>x \mid \eta(y)\}=\mathbf{P}\left\{Z^{\prime}>x-y\right\}=P(x-y)$

$$
P(x)=P(x-y) \mathbf{P}\{\eta(y)<\infty\}=P(y) P(x-y) .
$$

Решения этого уравнения в классе монотонно убывающих функций имеют вид

$$
P(x)=e^{-b x}, \quad b \geqslant 0 .
$$

Так как $\mathbf{P}\{Z>x\} \rightarrow 0$ при $x \rightarrow \infty$, то $b>0$ и выполнено (26). 
Пусть теперь выполнено (26). Допустим, что процесс $Y(\cdot)$ имеет положительные скачки и, стало быть, $\mathbf{P}\{\chi(y)>0\}>0$. Тогда в силу (26)-(28)

$$
\mathbf{P}\left\{Z^{\prime}>x-y-\chi(y)\right\}>P(x-y), \quad P(x)>P(y) P(x-y) .
$$

Это противоречит (26).

Теорема 7 доказана.

\section{СПИСОК ЛИТЕРАТУРЫ}

1. Kingman J.F.C. On queues in heavy traffic. - J. Roy. Statist. Soc. Ser. B, 1962, v. 24, p. $383-392$.

2. Прохоров Ю.В. Переходные явления в процессах массового обслуживания. Литов. матем. сб., 1963, т. 1, с. 199-206.

3. Боровков $A$. A. Вероятностные процессы в теории массового обслуживания. М.: Наука, 1972, $367 \mathrm{c}$.

4. Cohen J. W. Random walk with a heavy-tailed jump distribution. - Queueing Syst., 2002 , v. 40 , № 1 , p. $35-73$.

5. Boxma O.J., Cohen J. W. Heavy-traffic analysis for the GI/G/1 queue with heavytailed distributions. - Queueing Systems Theory Appl., 1999, v. 33, № 1-3, p. 177204.

6. Cohen J. W. A heavy-traffic theorem for the GI/G/1 queue with Pareto-type service time distribution. - J. Appl. Math. Stochastic Anal., 1998, v. 11, № 3, p. 247-254.

7. Гнеденко Б.В., Колмогоров $A . H$. Предельные распределения для сумм независимых случайных величин. М.: Гостехиздат, 1949, 264 с.

8. Биллингсли П. Сходимость вероятностных мер. М.-Л.: Наука, 1977, 352 с.

9. Боровков $A$. A. Большие уклонения для случайных блужданий с разнораспределенными скачками, имеющими бесконечную дисперсию. - Сиб. матем. журн., 2005 , т. 46 , № 1 , c. $46-70$.

10. Градштейн И. С., Рыжик И. М. Таблицы интегралов, сумм, рядов и произведений. М.: Физматгиз, 1962. 